\title{
Water-Like Anomalies for Core-Softened Models of Fluids: Two-Dimensional Systems
}

\author{
A. Scala, M. Reza Sadr-Lahijany, N. Giovambattista, \\ S. V. Buldyrev, and H. E. Stanley \\ Center for Polymer Studies and Department of Physics \\ Boston University, Boston, MA 02215 USA
}

(12 October 2000)

\begin{abstract}
We use molecular dynamics simulations in two dimensions to investigate the possibility that a core-softened potential can reproduce static and dynamic anomalies found experimentally in liquid water: (i) the increase in specific volume upon cooling, (ii) the increase in isothermal compressibility upon cooling, and (iii) the increase in the diffusion coefficient with pressure. We relate these anomalies to the shape of the potential. We obtain the phase diagram of the system and identify two solid phases: a square crystal (high density phase), and a triangular crystal (low density phase). We also discuss the relation between the anomalies observed and the polymorphism of the solid. Finally, we compare the phase diagram of our model system with experimental data, noting especially the line of temperatures of maximum density, line of pressures of maximum diffusion constant, and line of temperatures of minimum isothermal compressibility.
\end{abstract}

PACS numbers: 61.20.Gy, 61.25.Em, 65.70.+y, 64.70.Ja 


\section{INTRODUCTION}

Most liquids contract upon cooling and become more viscous with pressure. This is not the case for the most important liquid on earth, water. For at least 300 years it has been known that the specific volume of water at ambient pressure starts to increase when cooled below $T=4^{\circ} \mathrm{C}[1]$. It is perhaps less known that the viscosity of water decreases upon increasing pressure in a certain range of temperatures [2]. Moreover, in a certain range of pressures water exhibits an anomalous increase of compressibility, and hence of density fluctuations, upon cooling. These anomalies are not restricted to water but are also present in other liquids 3 [5.

In order to investigate these anomalies, we utilize computer simulation of a class of potentials called "core softened" potentials, first introduced by Stell, Hemmer, and their coworkers [6]. We define a core-softened potential as a spherically symmetric potential that has a region of negative curvature in its repulsive core [7]. An example of a discrete and of a smooth core-softened potential is shown in Fig. 1. Debenedetti et al. noted that a "softened core" can lead to a density anomaly [8], i.e., one of the anomalies found in water. Furthermore, ab initio calculation [9] and inversion of the experimental oxygen-oxygen radial distribution function reveals that a "core-softened" potential can be considered a realistic first-order approximation for the interaction between water molecules [10].

Although directional bonding is certainly a fundamental issue in obtaining quantitative predictions for network-forming liquids like water, it could be the case that core-softened potentials can be the simplest framework to understand the physics of those anomalies. Here we demonstrate, by means of numerical simulations for $d=2$, that the core-softened potential can lead to anomalies in the density, in the compressibility and in the viscosity. We also offer an explanation for the occurrence of these three anomalies in terms of the shape of the potential.

The paper is organized as follows: in Sec. [1] we define the potentials studied and in Sec. III we describe the methods of simulations employed. In Sec. $\mathbb{\square}$ we present the results 
about the density anomaly. In Sec. $\square$ we discuss the relation of the structures in the solid phase and the density anomalies. In Sec. VI we present the results for the diffusion anomaly and give an explanation of such anomaly in terms of free volume. In Sec. VII we present the results on the compressibility anomaly. Finally, we present the overall phase diagram in Sec. VIII and our conclusions and comments in Sec. IX.

\section{DISCRETE AND SMOOTH MODELS}

\section{A. Discrete Potential}

The core-softened potentials that we study are shown in Fig. 1 as a function of particle pair distance $r$. The discrete potential is composed of a hard core of diameter $a$ which has a repulsive shoulder of width $b-a$ at depth $\lambda \epsilon$, and an attractive well of width $c-b$ and depth $\epsilon$. The form of the function is thus

$$
u(r)= \begin{cases}\infty & 0<r<a \\ -\lambda \epsilon & a<r<b \\ -\epsilon & b<r<c \\ 0 & r>c\end{cases}
$$

All of the results reported here for the discrete potential are for $a=1, b=\sqrt{2}, c=\sqrt{3}$, $\epsilon=2$, and $\lambda=0.5$.

In the case of water, one can attribute the larger distance $r=b$ to hydrogen bonding, for which the system acquires a low energy and expands at the same time. The inner distance $r=a$ on the other hand corresponds to a non-hydrogen bonded energy state. Recent studies have proposed this form of potential as the interaction between clusters of strongly bonded pentamers of water [11]. This type of interaction is expected to reproduce the density anomaly. The reason is that at low pressures and at low temperatures, nearest neighbor pairs sit in the outer well which has a lower energy. By increasing $T$, in order to gain more entropy, the system explores a larger portion of the configurational space, which 
is not probed at lower temperatures. This includes penetrating the "soft" core $(a<r<b)$, which on average can lead to anomalous contraction upon heating.

\section{B. Smooth Potential}

While the discrete potential $u(r)$ is appropriate for deriving the closed form of equation of state in $1 d$ [5, 12, 13], for simulations it is not necessarily the most appropriate. As we will show in the next section, the smooth version of the potential $u^{\prime}(r)$ requires a different

method of simulation from that of $u(r)$. The potential $u^{\prime}(r)$ we use is obtained by adding a Gaussian well to the Lennard-Jones potential and has the form

$$
u^{\prime}(r)=4 \epsilon^{\prime}\left[\left(\frac{\sigma}{r}\right) r^{12}-\left(\frac{\sigma}{r}\right) r^{6}\right]-\lambda^{\prime} \epsilon^{\prime} \exp \left[-w^{n}\left(\frac{r}{\sigma}-\frac{r_{0}}{\sigma}\right)^{n}\right],
$$

for $r \leq r_{c}$ and vanishes for $r>r_{c}$. We use $\epsilon^{\prime}=1.0, \lambda^{\prime}=1.7, w=5.0, r_{0}=1.5, \sigma=1, n=2$ in order to mimic the shape of the discrete potential, as shown in Fig. 1.

\section{MOLECULAR DYNAMICS SIMULATION METHODS}

The method of simulation in both the discrete and the smooth cases is the molecular dynamics (MD) method. Our simulations are performed in $2 d$ with periodic boundary conditions. The overall qualitative results of the simulations for the discrete and the smooth potential are similar, while the quantitative results differ. In what follows, we explain in more detail the MD method used in each case.

\section{A. Constant-Volume Simulation of the Discrete Potential}

For the discrete potential [Eq. (11)], we use the collision table technique [14] for $N=896$ disks. To each disk we assign a radius $a / 2$. We define the density $\rho$ to be the ratio of the total area of all the disks to the area of the box. Energies are measured in units of $\epsilon$, temperature is calculated in units of energy divided by the Boltzmann constant, $\epsilon / k_{B}$, and the mass of the particle is set at $m=1$. 
The average pressure is calculated using the virial equation for step potentials [14]

$$
P=\left\langle\frac{1}{V}\left[K+\frac{1}{2 \delta t} \sum_{i, j}^{\prime} m\left(\vec{v}_{i}^{\prime}-\vec{v}_{i}\right)\left(\vec{r}_{i}-\vec{r}_{j}\right)\right]\right\rangle,
$$

where $K=\sum_{i} m \vec{v}_{i}^{2} / 2$ is the total kinetic energy, $N$ is the number of particles, $\sum_{i, j}^{\prime}$ is the sum over the particle pairs $(i, j)$ undergoing a collision in the time interval $\delta t, \vec{v}_{i}$ and $\vec{v}_{i}^{\prime}$ are the velocities of the particle $i$ before and after a collision, $\vec{r}_{i}$ and $\vec{r}_{j}$ are the positions of the particles $i$ and $j$ undergoing a collision at the start of $\delta t$.

We simulate state points along constant-volume paths. For thermalization we use the Berendsen method of rescaling the kinetic energy [14]. We thermalize the system for $10^{5}$ time units, which corresponds to $\sim 10^{6}$ collisions per particle, and then acquire data for $10^{6}$ time units corresponding to $\sim 10^{7}$ collisions per particle.

\section{B. Constant-Volume Simulation of the Smooth Potential}

For the smooth version of the potential [Eq. (2)], we use the velocity Verlet integrator method [14] for a system of $N=2500$ discs. We record the results in reduced units in which $\sigma, \epsilon^{\prime}, m$, and $k_{B}$ are all unity. We choose $r_{c}=2.5$, and the length of each MD time step $\delta t=0.01$. We assign to each particle a radius $2^{1 / 6}$, which corresponds to the minimum of the Lennard-Jones potential $\sigma$, and define the density $\rho$ to be the ratio of the total area of all the disks to the area of the box.

In order to achieve a preset temperature we use the Berendsen method of rescaling the velocities [14], resulting in the time dependence

$$
T(t)=T_{\infty}+\left(T(0)-T_{\infty}\right) e^{-t / \tau}
$$

where $\tau$ is a preset time constant [14]. Typical values of $\tau$ are around $10^{4} \delta t$.

We first thermalize the system for a time $\Delta t \approx 10 \tau$, and we ensure that equilibrium is attained by monitoring the time dependence of observables like $T, P$ and the potential energy $U$. Then we acquire data, running the system for an additional period of time at 
constant NVE conditions (micro-canonical ensemble). We calculate $P$ and $T$ and we consider the system to be in equilibrium only when the fluctuations of these quantities are less than $1 \%$ of their average values. The acquisition time is chosen to be more than the time it takes for the system to equilibrate and is typically $\left(5 \times 10^{4}\right) \delta t$ to $\left(2 \times 10^{5}\right) \delta t$.

The average simulation speed on Boston University's SGI Origin 2000 supercomputer was approximately $10 \mu s$ per particle update. Each of the state points we study requires between 8 and 16 hours on one processor, and thus over 1000 hours total computational time was utilized.

\section{Constant-Pressure Simulation of the Smooth Potential}

In order to check that our results are not biased by problems like phase coexistence that can affect constant-volume simulations, we also perform constant-pressure simulations in the case of the smooth potential. Constant-pressure simulations allow us to determine more accurately the locations of the freezing line and the density maximum. We use the feedback

method proposed by Broughton [15], where the dimensions of the box and the positions of the particles are rescaled to obtain the desired pressure $P$. The amount of rescaling depends on the difference between the present pressure $P(t)$ and the desired pressure $P$.

Using the Broughton method and the Berendsen method, we gradually drive the system to the desired $P$ and $T$, while simulating under readjusting $V$ and $E$ conditions. We choose pressure and temperature tolerances $\delta P$ and $\delta T$ less than $1 \%$ of the desired $P$ and $T$. Once $P(t)$ and $T(t)$ reach values within the range $P \pm \delta P$ and $T \pm \delta T$, we stop thermalization and pressurization. If the system stays within these limits for an interval of time of the same order of time needed to reach the desired $P$ and $T$, we conclude that the system has equilibrated, turn off the thermalization and pressurization, and start collecting data. During this collection period, we monitor temperature and pressure to check that their average values coincide with the desired ones within an accuracy of $1 \%$. For our results, the time $\Delta t$ needed to reach equilibrium is usually of the order of 500,000 steps $\delta t$, so 
$\Delta t \approx 5000$ in Lennard-Jones units. Data are acquired over a period of $10 \Delta t$. We test our code by simulating a Lennard-Jones system of 2304 disks and comparing the results with the previously-known phase diagram of a $2 d$ Lennard-Jones system.

\section{DENSITY ANOMALY}

The temperature of density maximum $\left(T_{M d}\right)$ line is the border of the region in the $P-T$ plane where the liquid expands upon cooling. Fig. 2 shows a set of different isobars for the smooth potential. The $T_{M d}$ line corresponds to the set of maxima along those isobars.

In the case of constant-volume simulations, the $T_{M d}$ line corresponds to the minima along constant-volume paths ( $P$ vs. $T$ graphs of Fig. 3 ) since for any thermodynamic quantity $X$ constant-volume paths

$$
\left(\frac{\partial X}{\partial T}\right)_{V}=\left(\frac{\partial X}{\partial T}\right)_{P}-\frac{(\partial X / \partial P)_{T}(\partial V / \partial T)_{P}}{(\partial V / \partial P)_{T}}
$$

By substituting $X=P$ we find

$$
\left(\frac{\partial P}{\partial T}\right)_{V}=\frac{\alpha_{P}}{K_{T}}
$$

where

$$
\alpha_{P} \equiv V^{-1}\left(\frac{\partial V}{\partial T}\right)_{P}
$$

is the thermal expansion coefficient and

$$
K_{T} \equiv-V^{-1}\left(\frac{\partial V}{\partial P}\right)_{T}
$$

is the isothermal compressibility. Taking a derivative of Eq. (5), using $\alpha_{P}=0$ at the $T_{m d}$ line, we find

$$
\left(\frac{\partial^{2} P}{\partial T^{2}}\right)_{V}=\frac{V^{-1}\left(\partial^{2} V / \partial T^{2}\right)_{P}}{K_{T}}
$$

Equations (6) and (9) show that since $K_{T}$ is always positive and finite for systems in equilibrium not at a critical point, a minimum along the isochore is equivalent to a minimum 
along an isobar, which is the density maximum point $T_{M d}$. Figure 3 shows the isochores for the smooth and the discrete potentials.

To confirm that we are investigating the liquid state part of the phase diagram, we introduce a criterion to distinguish the liquid state from a frozen state. We determine the freezing line as the location of points where isochores overlap. In this way we establish an approximate location for the freezing line. Crossing this line from the liquid side, we find a sharp decrease in $D$ coinciding with the appearance of slowly-decaying peaks in $g(r)$ as a function of $r$, which signals the build up of long-range correlations (Fig. đ), which is a characteristic of $2 \mathrm{D}$ solids.

We confirm the above criterion adopted to locate the freezing lines by using isobaric simulations for the smooth potential. Indeed, they show a sharp change of density, in correspondence with the estimated freezing line at high pressures (Fig. 5, lower panel). The presence of a hysteresis loop (Fig. 6) suggests that the liquid-solid transition is first-order; however, by lowering the pressure the loops become less and less pronounced and eventually disappear, leaving the possibility of an hexatic second-order transition [16].

For a few state points near the freezing line, we have checked our results by simulating $N=2500$ particles in rectangular boxes with aspect ratio $\sqrt{3} / 2\left(L_{x}=\sqrt{3} / 2 L_{y}\right.$, with $L_{x} \times L_{y} \equiv L_{0}^{2}$ ) which accommodate triangular lattices perfectly. This eliminates any possible artificial hindrance in crystallization that may arise from the asymmetry imposed by the shape of the square box.

For water, the locus of the $T_{M d}$ line in the P-T phase diagram is of special interest to distinguish between different scenarios proposed to explain its anomalies [17 19]. In Fig. [7 we see that the $T_{M d}$ line changes from negative slope at high pressures to positive slope at low pressures. This change in slope is similar to what is observed in simulating model potentials of water like SPC/E or ST2 [17, 20]. These results suggest that the change in slope can be a general phenomenon stemming from the general core-softened form of the interaction in the simulation. 


\section{STRUCTURES IN THE LIQUID AND SOLID PHASES}

Figure 5 (upper panel) shows the different phases of the system. In our simulations we see that the $T_{M d}$ line is located in the region of pressures where the freezing line is negatively sloped, as in water. A density anomaly and a negatively-sloped melting line are often associated [3, 21]. This has proven to be the case for substances like water (Fig. 8) and tellurium [5] and for computer models [12, 22]. This association is plausible since the isobaric thermal expansion coefficient $\alpha_{P}$ is related to the cross fluctuations in volume and entropy as

$$
\alpha_{P} \equiv \beta\langle\delta V \delta S\rangle
$$

Approaching a freezing line, we expect local density fluctuations to have structures similar to the neighboring solid as they are going to trigger the liquid-solid transition. On the other hand, the Clausius-Clapeyron relation for the slope of the freezing line

$$
\frac{d P}{d T}=\frac{\Delta S}{\Delta V}
$$

implies that, if the freezing line is negatively sloped, the solid, which has a lower entropy than the liquid, will have a higher specific volume. Therefore, if the fluctuations in the liquid are "solid-like," $\alpha_{P}[$ Eq. (10)] will turn out to be negative.

To distinguish different local structures in the liquid, we plot the radial distribution function $g(r)$ for different pressures and temperatures (Fig. 团). As expected, at low pressures cooling expels particles from the core, while increasing pressure at fixed temperature has the opposite effect.

Since our system is two-dimensional, we can use visual inspection to develop an intuitive picture of the possible local structures (Fig. 5, upper panel). If the fluctuations in the liquid are "solid-like," near the freezing line we expect to see local structures that resemble the structure of the nearby solid.

We find that at low $P$ and $T$, the system is frozen with a hexagonal structure (Fig. 9, lower left panel). A "snapshot" of the system along the same isobaric line (Fig. 9, lower right 
panel) shows clearly that local patches with hexagonal order are present in the liquid phase near the freezing line. We will refer to this structure as the "open structure." Similarly, at high pressures the local patches in the liquid phase near the freezing line (Fig. 9, upper right panel) resemble the structure of the system when it is frozen at low $T$ and high $P$ (Fig. 9, upper left panel). We will refer to this as the "dense structure." For the open structures, each particle has six neighbors sitting in the deepest well, and the softened-core behaves as the effective core for the particles. The dense structure is the next energetically favorable local arrangement, with four neighbors in the external well and four in the softened core, for which the effective core is the hard core.

\section{DIFFUSION ANOMALY}

We next study the diffusion anomaly, which is another surprising feature of water. While for most materials diffusivity decreases with pressure, liquid water has an opposite behavior in a large region of the phase diagram [2] (Fig. 8). The pressures where the system has a maximum diffusivity along isotherms define the line of the pressure of maximum diffusivity, $P_{M D}$

We observe that our core-softened potential reproduces this anomaly. We first measure the mean square displacement $\left\langle\Delta r^{2}(t)\right\rangle \equiv\left\langle\left[r\left(t+t_{0}\right)-r\left(t_{0}\right)\right]^{2}\right\rangle$ and then the diffusion coefficient using the relation

$$
D=\frac{1}{2 d} \lim _{t \rightarrow \infty} \frac{\left\langle\Delta r^{2}(t)\right\rangle}{t}
$$

We measure $\left\langle\Delta r^{2}(t)\right\rangle$ by averaging over the starting time $t_{0}$ in Eq. (12). We find that there is a region of the phase diagram in which $D$ increases upon increasing $P$ (Fig 10).

In order to understand the diffusion anomaly we first note that, for normal liquids, $D$ decreases with $P$ because upon increasing $P$ the density increases and molecules are more

constrained. As a result the viscosity increases and hence $D$ decreases. In the case of water the anomaly can be related to the fact that increasing pressure (and hence density) 
breaks hydrogen bonds, which in turn increases the mobility of the molecules. We present a more general explanation which can equally apply to our radially symmetric core-softened interaction which does not possess any directional bonds similar to hydrogen bonds. The low energy inter-particle state at $r \approx b$ plays the role of non-directional bond. Note that $D$ is proportional to the mean free path of particles, which increases with the free volume per particle $v_{\text {free }} \equiv v-v_{\text {ex }}$, where $v_{\text {ex }}$ is the excluded volume per particle resulting from the effective hard core. At low temperatures, $v_{\mathrm{ex}}$ for the dense structure is proportional to the area $a^{2}$ of the hard core, while for the open structure it is proportional to the area $b^{2}$ of the soft core. Increasing $P$ decreases $v$, which is the main effect in normal liquids. For the core-softened liquid, on the other hand, increasing $P$ can also decrease $v_{\text {ex }}$ by transforming some of local open structures to dense structures. Since both $\Delta v$ and $\Delta v_{\text {ex }}$ decrease with $P$ and since $\Delta v_{\text {free }}=\Delta v-\Delta v_{\mathrm{ex}}$, the effect of $P$ on $D$ depends on whether $\Delta v$ or $\Delta v_{\mathrm{ex}}$ dominates. The anomalous increase in $D$ along the isotherms near the freezing line is a sign of the dominance of the $\Delta v_{\mathrm{ex}}$ term. Thus the anomaly in $D$ must disappear near a certain pressure above which the average distance between particles corresponds to the dense structure, and as a result the contribution of the open structure to $v_{\mathrm{ex}}$ is negligible.

We verified this in our simulation by observing a correspondence between the disappearance of the diffusion anomaly and the disappearance of the peak in $g(r)$, corresponding to the open structure that is observed in real water.

\section{ISOTHERMAL COMPRESSIBILITY}

In order to investigate the anomaly in isothermal compressibility $K_{T}$, we calculate $K_{T}$ at each state point using the data in Fig. 3. In the smooth potential case we verify these results using

$$
K_{T}=\lim _{q \rightarrow 0} \frac{S(q)}{n k_{B} T}
$$

as an alternative method [23], where $n$ is the density of the system and $S(q)$ is the structure function and is related to the pair correlation function via 


$$
S(q)=1+n \int e^{i \mathbf{q} \cdot \mathbf{x}} g(\mathbf{x}) d \mathbf{x}
$$

We first calculate $g(r)$ for each state point averaging over all thermalized configurations. We then perform numerical integration using Eq. (14) to find $S(q)$, and finally we extrapolate $S(q)$ to $q=0$ and substitute in Eq. (13) to find $K_{T}$. We show an example of the graphs for $g(r)$ and the resulting $S(q)$ (normalized by the extra factors in Eq. 13) in Fig. 11. We fit the low $q$ tail of the curve to a line to find the limiting value in Eq. (13).

We graph $K_{T}$ along isobars, as shown in Fig. 12. For large $T$, the $K_{T}$ decreases upon increasing $P$. For small $T$ the behavior is the opposite and the compressibility anomaly occurs. As seen for all isobars shown in Fig. 12 (except a low pressure one), $K_{T}$ increases by lowering $T$.

\section{PHASE DIAGRAM}

In water the $T_{M d}$ line is negatively sloped for positive pressures. For several models that mimic water behavior, it is found that the $T_{M d}$ line has a reentrant shape, changing slope at low or negative pressures [3]. In our simulations, we find such a reentrant $T_{M d}$ line; the change of slope of the $T_{M d}$ happens at positive pressures in the smooth version and at negative pressures in the discrete case (Fig. 7).

Moreover, we have graphed the location of the minimum $K_{T}$ point along each isobar; the locus of these points is called the minimum compressibility line $\left(K_{T_{\min }}\right)$. Sastry and

coworkers [18], from basic thermodynamic arguments, show that: (i) the $K_{T_{\text {min }}}$ line intersects the $T_{M d}$ line at its infinite slope point, and (ii) the compressibility must increase upon cooling in the region to the left of a negatively sloped $T_{M d}$ line. Our results are in agreement with both of these statements (Fig. 7).

Theories relating $D$ to the entropy [24] predict that the anomalous behavior $(\partial D / \partial P)_{T}>$ 0 is related to an anomaly in the entropy $(\partial S / \partial P)_{T}>0$. Due to the Maxwell relation 
$(\partial S / \partial P)_{T}=-(\partial V / \partial T)_{P}$, whenever there is a density anomaly, an entropy anomaly occurs, and the value of entropy along isotherms reaches a maximum at the $T_{\mathrm{Md}}$ line.

In Fig. 7 we also show the $P_{M D}$ line where $D$ reaches its maximum with pressure. Notice that, for the continuous potential, the maximum in $D$ shifts to higher $P$ with increasing $T$. This trend is also observed in the $S P C / E$ model of water [25] but is in contrast with the behavior of real water (Fig. 8).

\section{SUMMARY}

We find that core-softened potentials reproduce the qualitative behavior of water in many respects; in particular, the liquid phase of core-softened potentials can show both thermodynamic anomalies and dynamic anomalies. Moreover, as in real water, the freezing line changes slope from a positive value at high pressures to a negative value at low pressures in the P-T phase diagram and more than one solid phase is present. The polymorphism of the solid phase and the anomalies in the liquid phase can be related to the possibility

of different local structures due to the shape of the potential. The phase diagrams of the discrete and the smooth versions of the core softened potential are similar to that of real water, but the $T_{M d}$ line is shifted into liquid phase and the $K_{T_{M I N}}$ line has a positive slope. However, only for the discrete potential we find a $P_{M D}$ line with a negative slope.

\section{ACKNOWLEDGEMENTS}

We thank D. Wolf, R. Speedy, F. Sciortino, M. Canpolat, F. W. Starr, E. La Nave, M. Meyer, A. Skibinsky and G. Stell for enlightening discussions, and NSF for support. 


\section{REFERENCES}

[1] Waller, R., trans. Essayes of natural experiments [original in Italian by the Secretary of the Academie del Cimento]. Facsimile of 1684 English translation (Johnson Reprint Corporation, New York, 1964).

[2] F. X. Prielmeier, E. W. Lang, R. J. Speedy, and H.-D. Lüdemann, Phys. Rev. Lett. 59, 1128 (1987); Ber. Bunsenges. Phys. Chem. 92, 1111 (1988); L. Haar, J. S. Gallagher, G. S. Kell, NBS/NRC Steam Tables. Thermodynamic and Transport Properties and Computer Programs for Vapor and Liquid States of Water in SI Units (Hemisphere Publishing Co., Washington DC, 1984), pp. 271-276.

[3] P. G. Debenedetti, Metastable Liquids (Princeton University Press, Princeton, 1996).

[4] O. Mishima and H. E. Stanley, Nature 396, 329 (1998).

[5] Y. Yoshimura, Ber. Bunsenges. Phys. Chem. 95, 135 (1991) and references therein.

[6] P. C. Hemmer and G. Stell, Phys. Rev. Lett. 24, 1284 (1970).

[7] J. S. Høye and P. C. Hemmer, Physica Norvegica 7, 1 (1973).

[8] P. G. Debenedetti, V. S. Raghavan and S. S. Borick, J. Phys. Chem. 95, 4540 (1991).

[9] K. K. Mon, N. W. Ashcroft, and G. V. Chester, Phys. Rev. B 19, 5103 (1979); J. Phys. F: Met. Phys. 15, 1215 (1985).

[10] T. Head-Gordon and F. H. Stillinger, J. Chem. Phys. 98, 3313 (1993).

[11] M. Canpolat, F. W. Starr, A. Scala, M. R. Sadr-Lahijany, O. Mishima, S. Havlin and H. E. Stanley, Chem. Phys. Lett. 294, 9 (1998).

[12] M. R. Sadr-Lahijany, A. Scala, S. V. Buldyrev and H. E. Stanley, Phys. Rev. Lett. 81 4895 (1998).

[13] M. Reza Sadr-Lahijany, A. Scala, S.V.Buldyrev, and H. E. Stanley, Phys. Rev. E 60, 
6714 (1999).

[14] M. P. Allen and D. J. Tildesley, Computer Simulation of Liquids (Oxford University Press, New York, 1989).

[15] J. Q. Broughton, G. H. Gilmer and J. D. Weeks, J. Chem. Phys. 75, 5128 (1981).

[16] D. R. Nelson, in Phase Transitions and Critical Phenomena, Vol. 7, edited by C. Domb \& J. L. Lebowitz (Academic, London, 1983), pp. 1.

[17] F. Sciortino, P. H. Poole, U. Essmann and H. E. Stanley, Phys. Rev. E 55, 727 (1997).

[18] S. Sastry, P. G. Debenedetti, F. Sciortino and H. E. Stanley, Phys. Rev. E 53, 6144 (1996).

[19] R. J. Speedy, J. Phys. Chem. 86, 982 (1982).

[20] S. Harrington, R. Zhang, P. H. Poole, F, Sciortino, and H. E. Stanley, Phys. Rev. Lett. 78, 2409 (1997); S. Harrington, P.H. Poole, F. Sciortino, H. E. Stanley, J. Chem. Phys. 107, $7443(1997)$.

[21] A. Scala, M. Reza Sadr-Lahijany, N. Giovambattista, S. V. Buldyrev, and H. E. Stanley, [Festschrift for G. S. Stell], J. Stat. Phys. 100, 97 (2000).

[22] E. A. Jagla, Phys. Rev. E 58, 1478 (1998).

[23] P. M. Chaikin and T. C. Lubensky, Principles of Condensed Matter Physics (Cambridge University Press, Cambridge, 1995).

[24] G. Adam and J. H. Gibbs, J. Chem. Phys. 43, 139 (1965).

[25] A. Scala, F. W. Starr, F. Sciortino, E. La Nave, and H. E. Stanley, Nature 406, 166 (2000). 


\section{FIGURES}

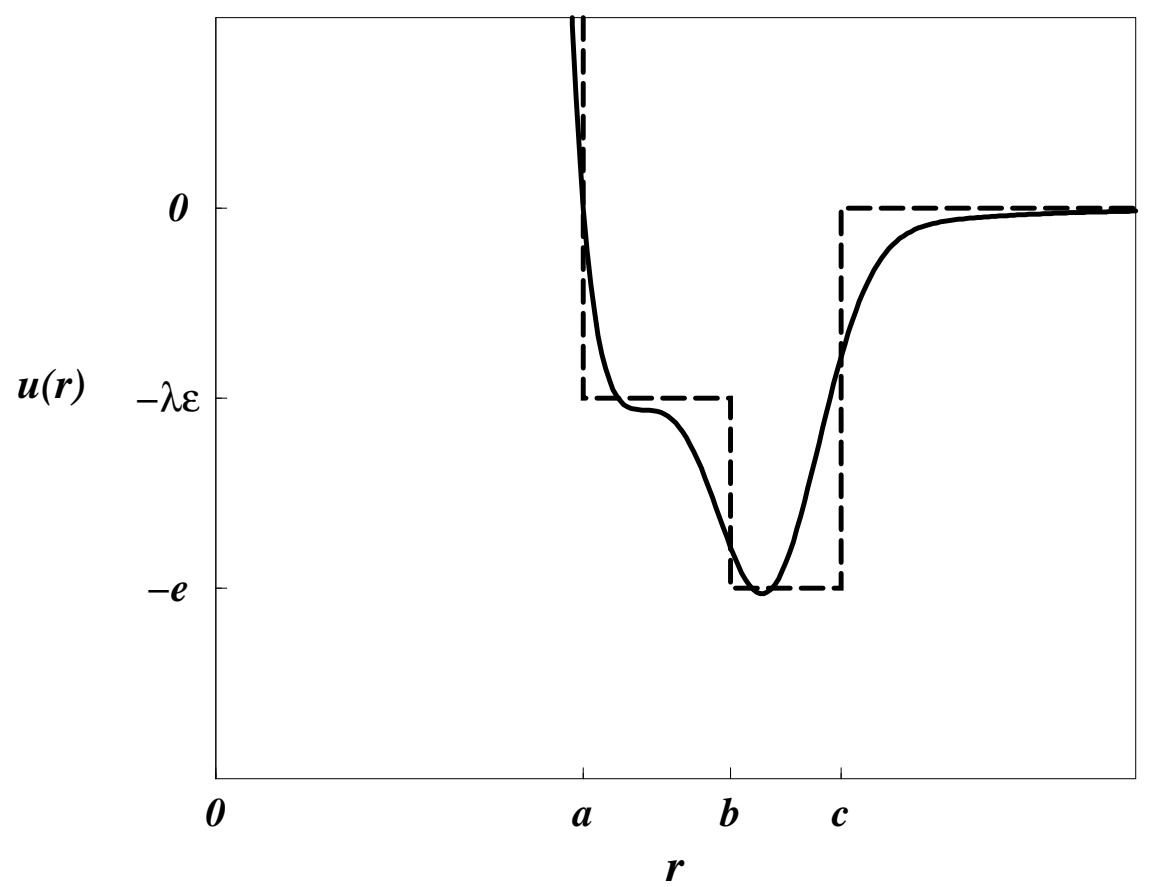

FIG. 1. Discrete (broken line) and smooth (solid line) forms of the core-softened potential $u(r)$ studied here. Length parameters $a, b, c$ and energy parameters $\epsilon, \lambda$ are shown. 


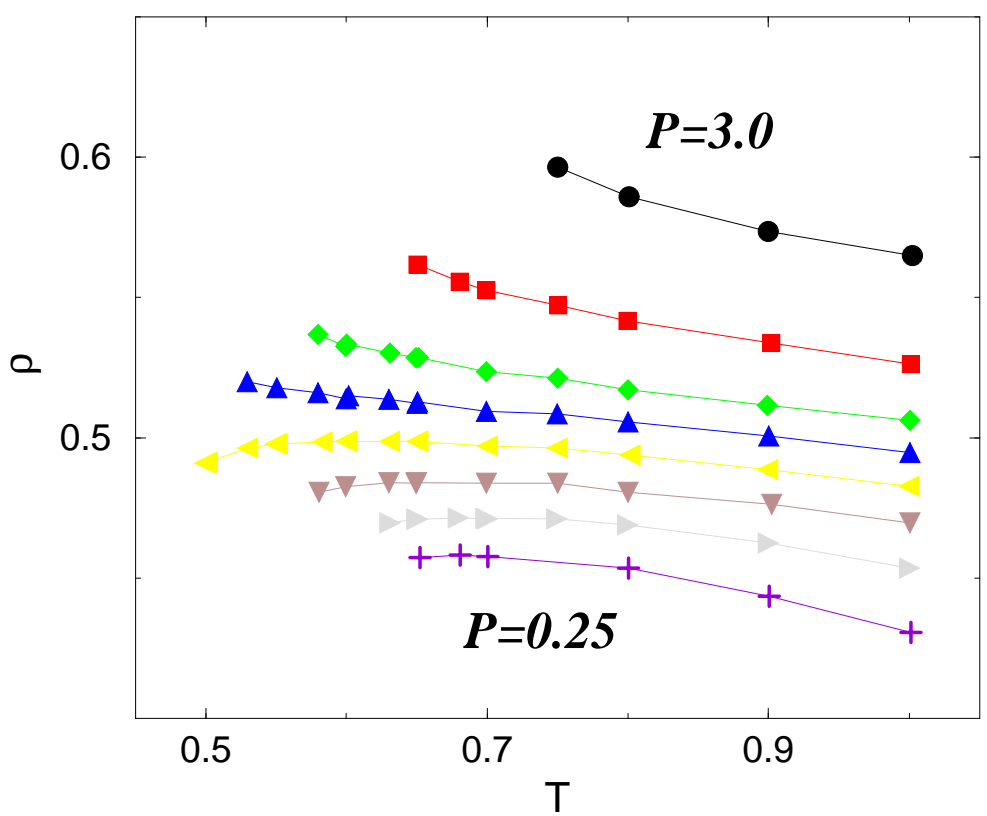

FIG. 2. Isobars for the smooth potential 

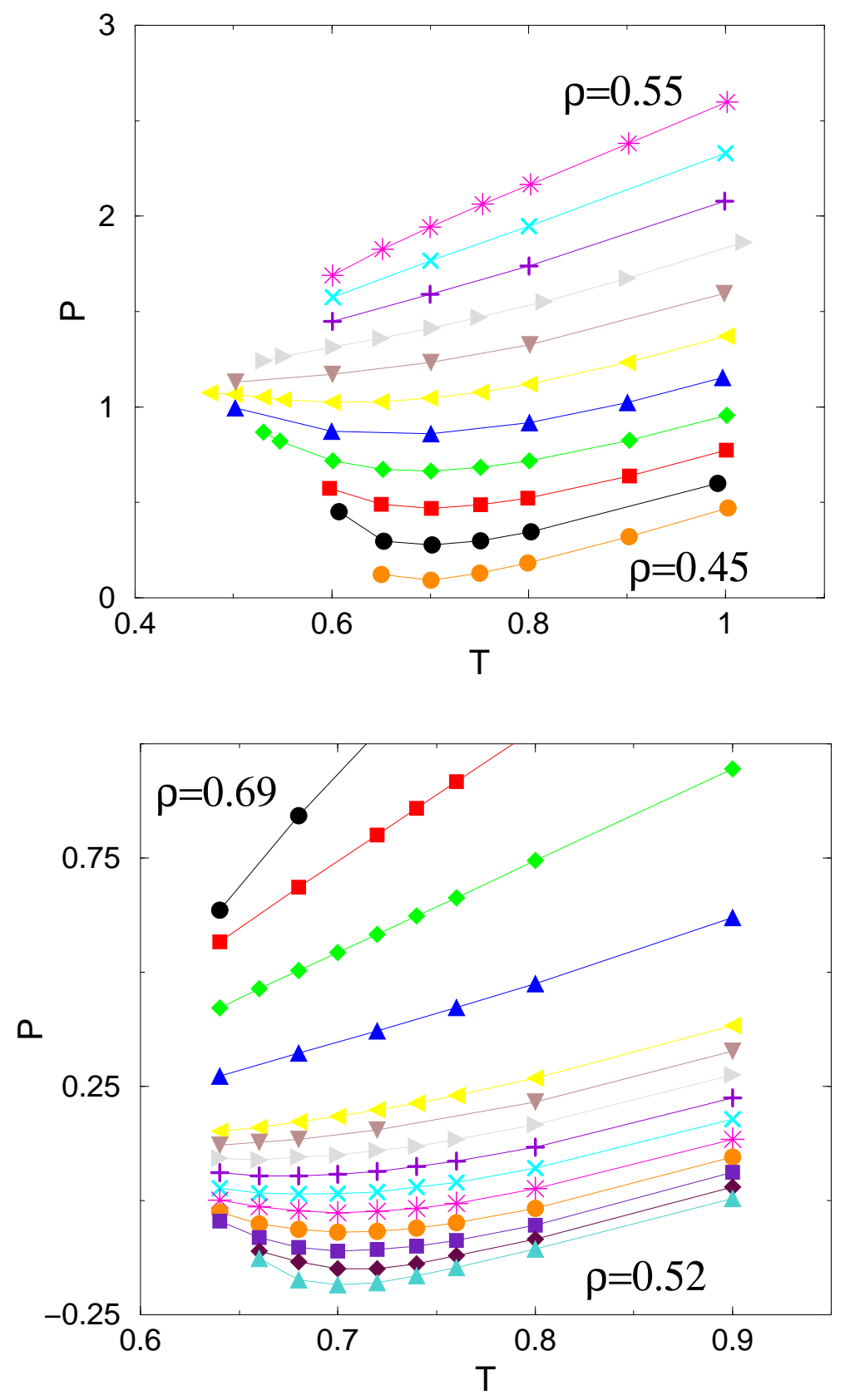

FIG. 3. Isochores for the smooth potential (left panel) and the discrete potential (right panel). 


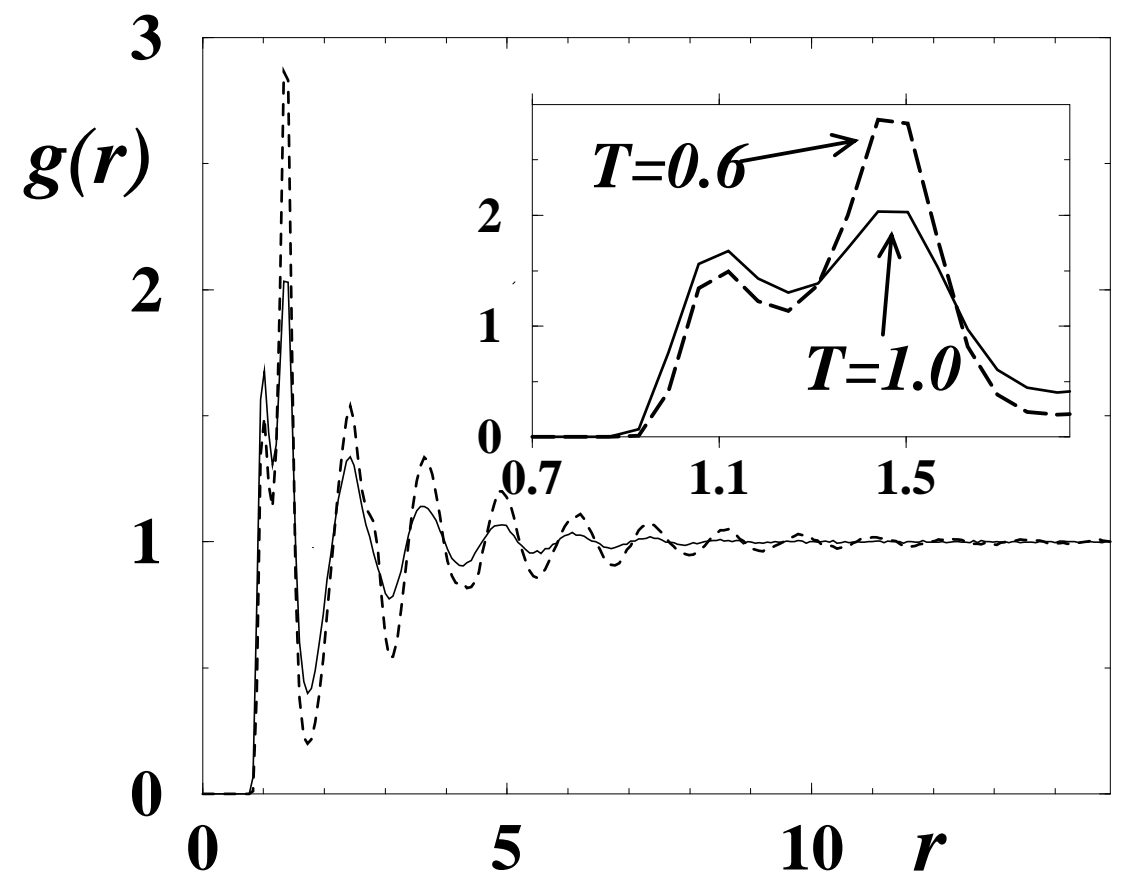

FIG. 4. Radial distribution function at high and low temperatures, along the $\mathrm{P}=0.48$ isobar for the smooth potential. Notice how, by lowering $T$, long range correlations develop $(g(r)=1$ if particles at distance $r$ are uncorrelated) and more particles are expelled from inside the soft core $r \sim 1.1$ into the attractive well $r \sim 1.5$ (inset). As the average interparticle distance is growing upon cooling, the system is expanding and there is hence a density anomaly. 

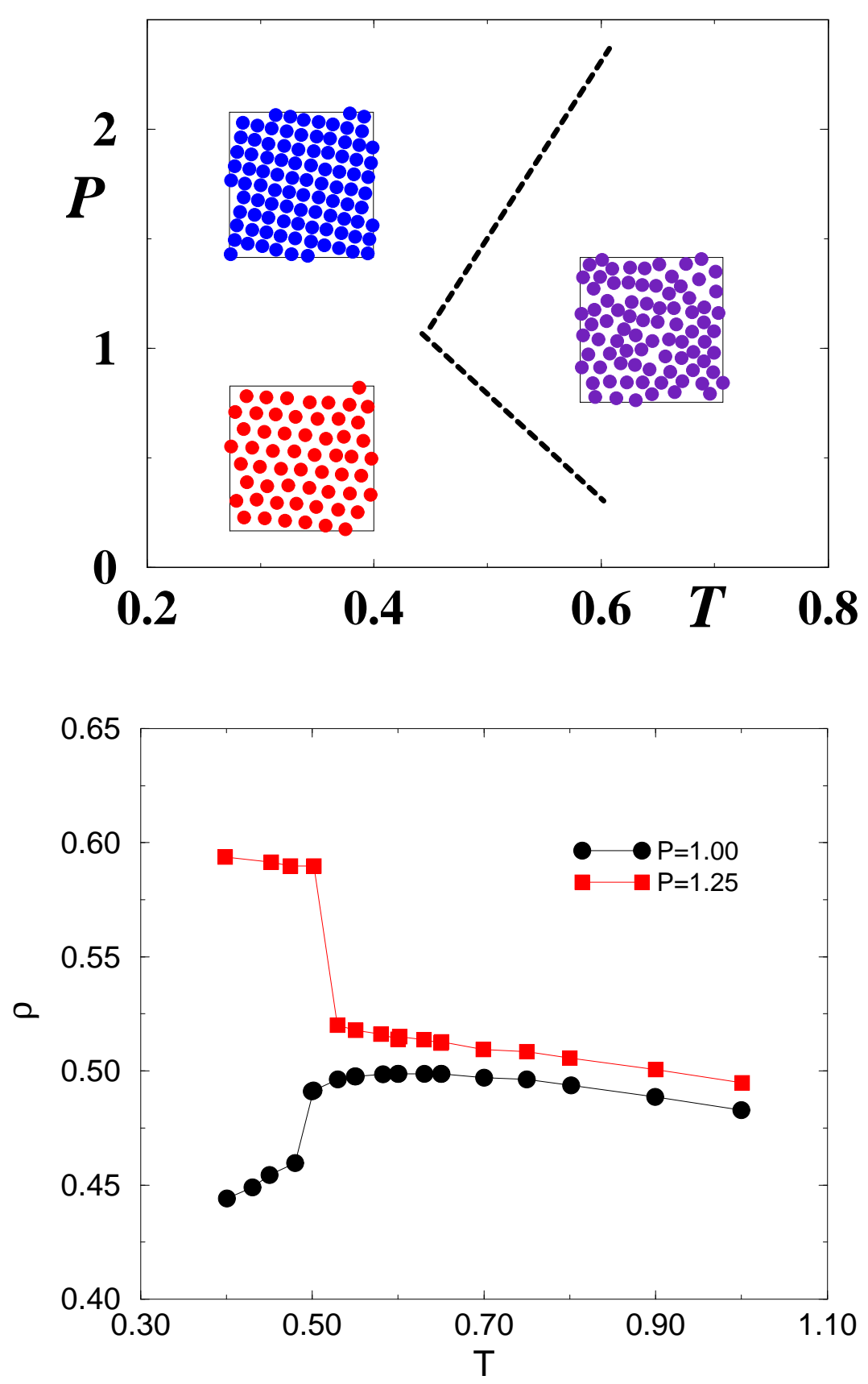

FIG. 5. Phases for the core-softened model (smooth potential). The upper panel shows a snapshot of the liquid phase and snapshots of different types of crystals for the solid phase at low pressures where the freezing line is negatively sloped, and at high pressures where the freezing line is positively sloped. Lower panel shows the density jumps along isobars; note that the low pressure isobar shows a density anomaly before jumping to a low density solid. 


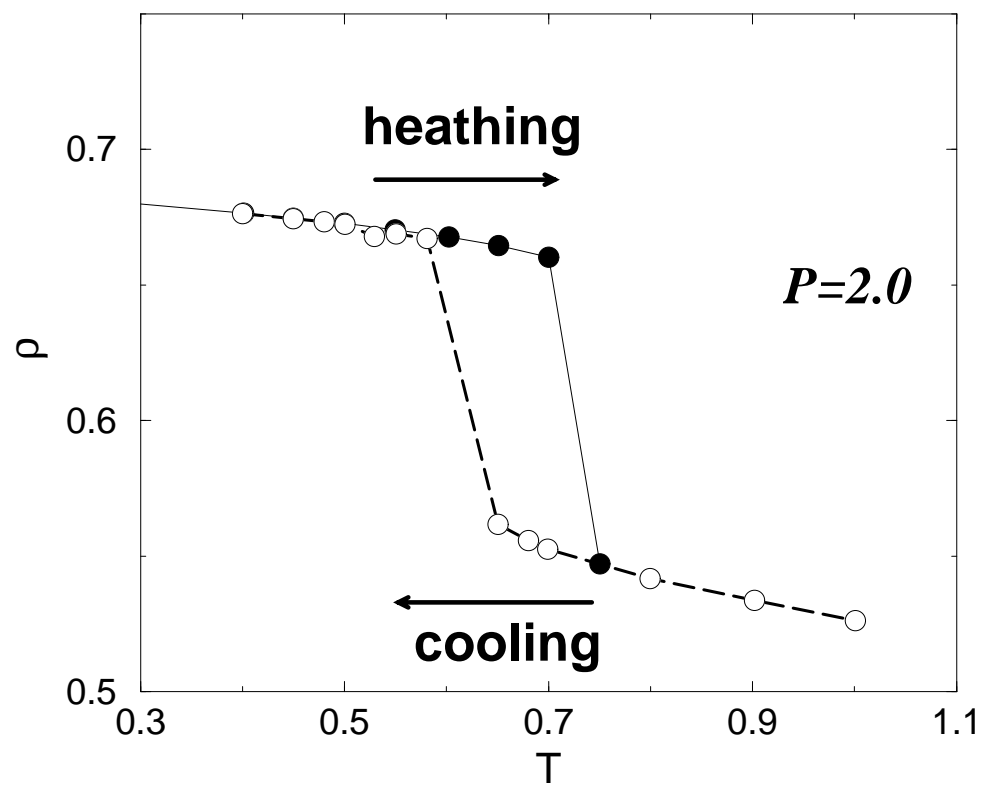

FIG. 6. Hysteresis loop near the freezing line for a high pressure isobar (smooth potential). The continuous line is obtained upon heating, the dashed line upon cooling. 

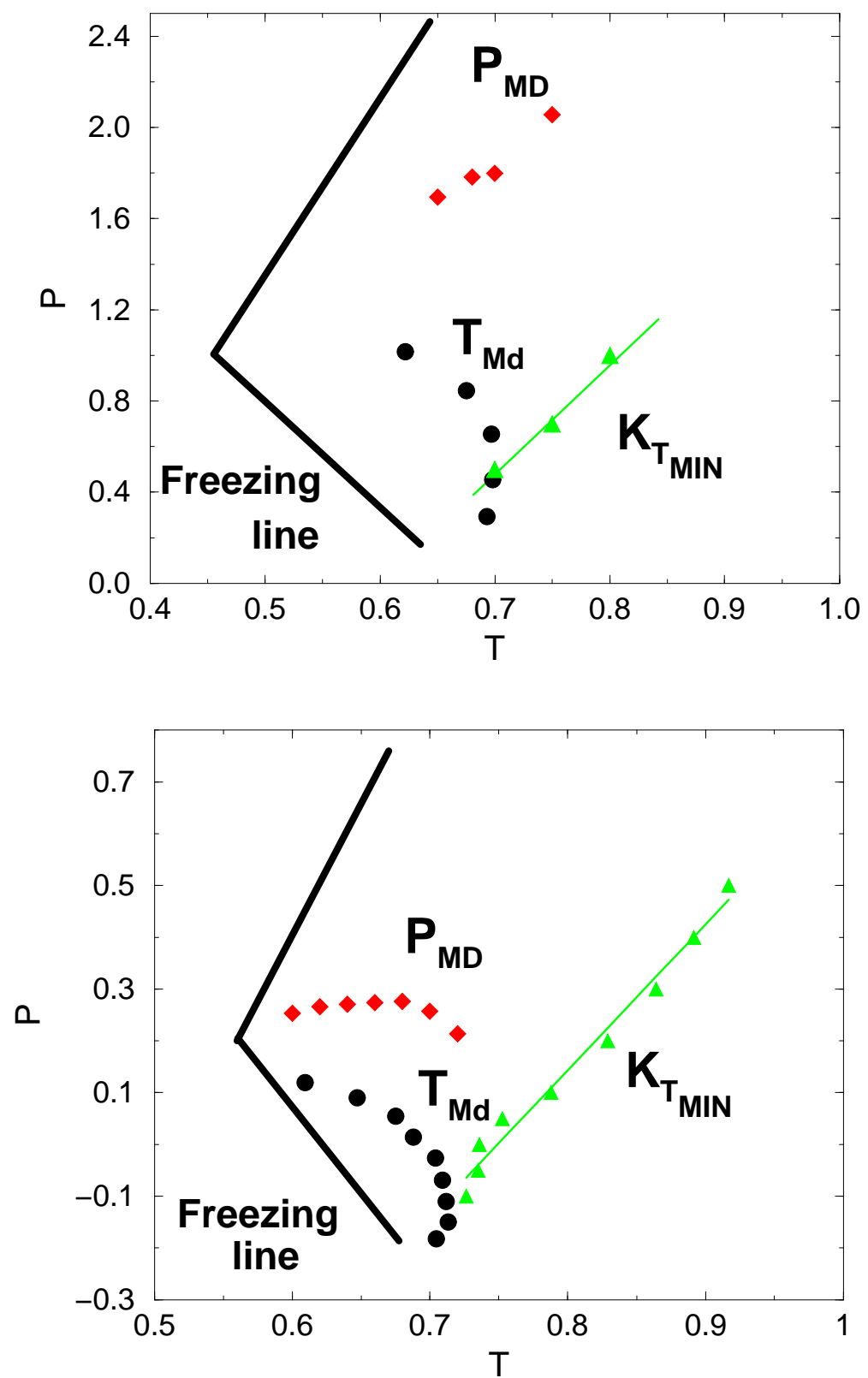

FIG. 7. Phase diagram with the $T_{M d}, P_{M D}, K_{T_{M I N}}$ and freezing line for the smooth potential (left panel) and discrete potential (right panel). 


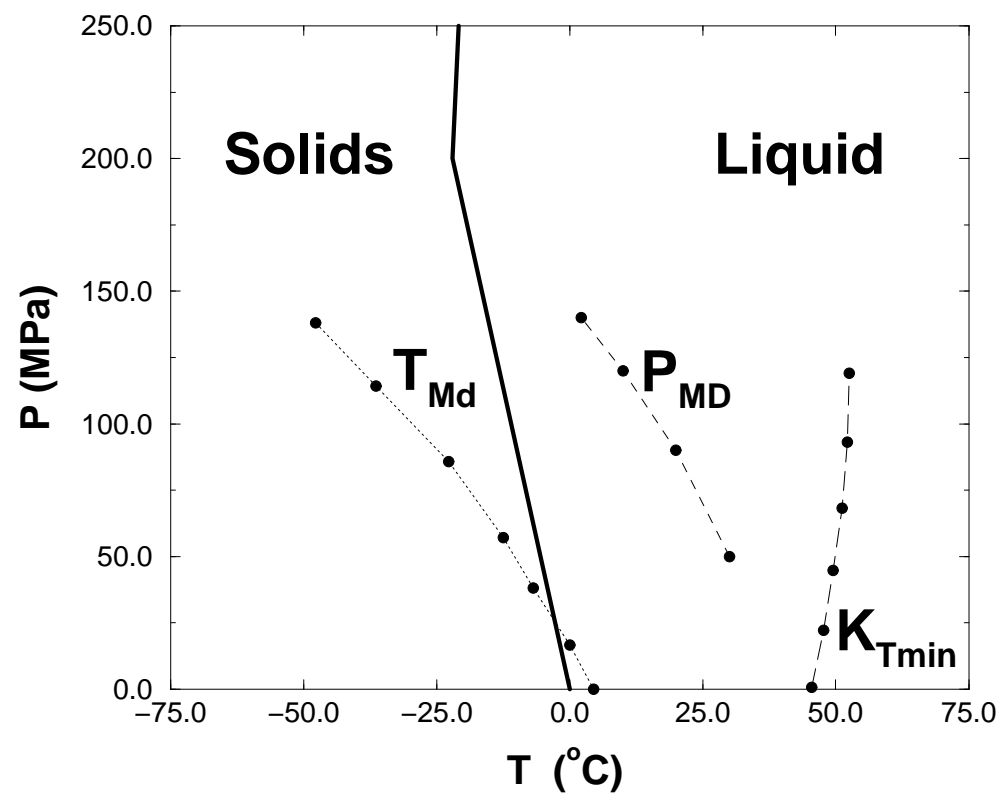

FIG. 8. Sketch of the phase diagram of water. The portion of the $T_{\mathrm{Md}}$ line that is to the left of the melting line corresponds to experiments in the supercooled region of water. Notice that the presence of a density anomaly in the region of the negatively sloped melting line can occur in the metastable phase of the liquid. Data are obtained from Refs. [2]. 

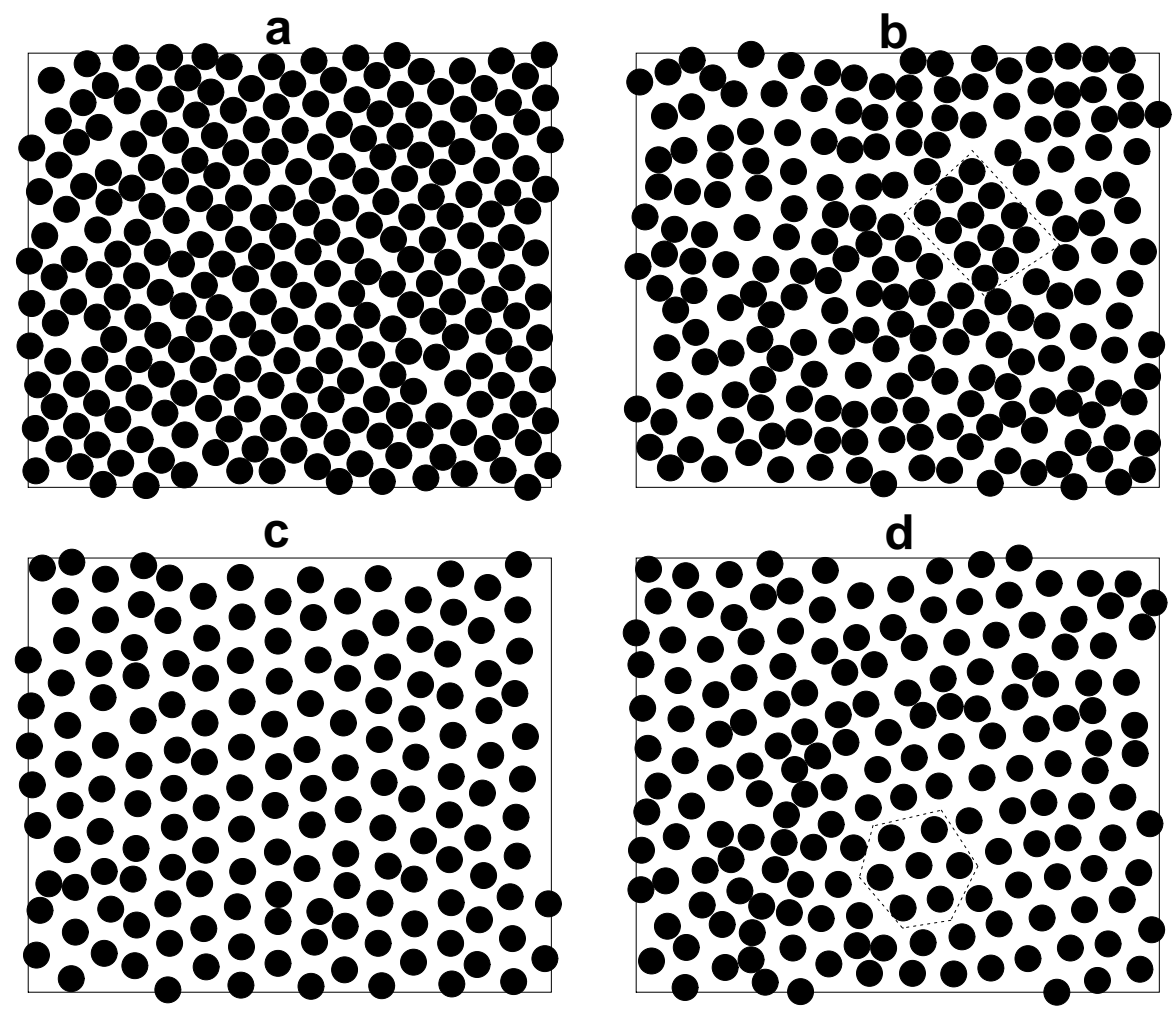

FIG. 9. Snapshots of the system (discrete potential) in the solid phase at high pressure (upper left) and low pressure (lower left), and in the liquid phase at high pressure (upper right) and low pressure (lower right). Moving along an isobar, patches of local order similar to the low temperature solid develop. At high pressure, the average distance between particles (which is the radius of the disk) is of the order of the hard core, while in the low pressure solid, the distance between particles is larger, of the order of the softened core. 

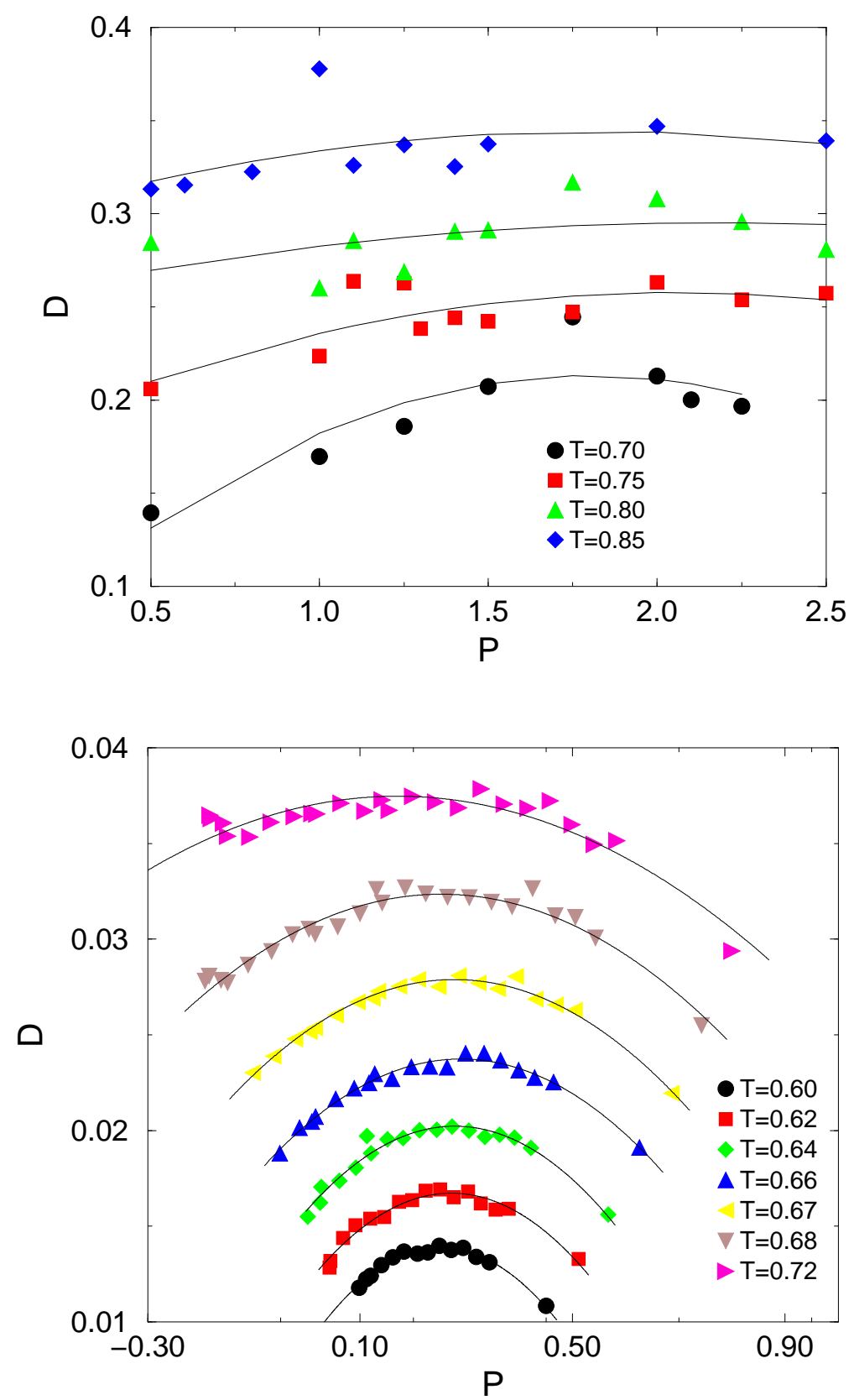

FIG. 10. Diffusion coefficient $D$ in the liquid phase for the continuous potential (left panel), the discrete potential (right panel) along various isotherms. Lines are intended as a guide for the eye. Notice the anomalous sections of the graph, where $(\partial D / \partial P)_{T}>0$. 

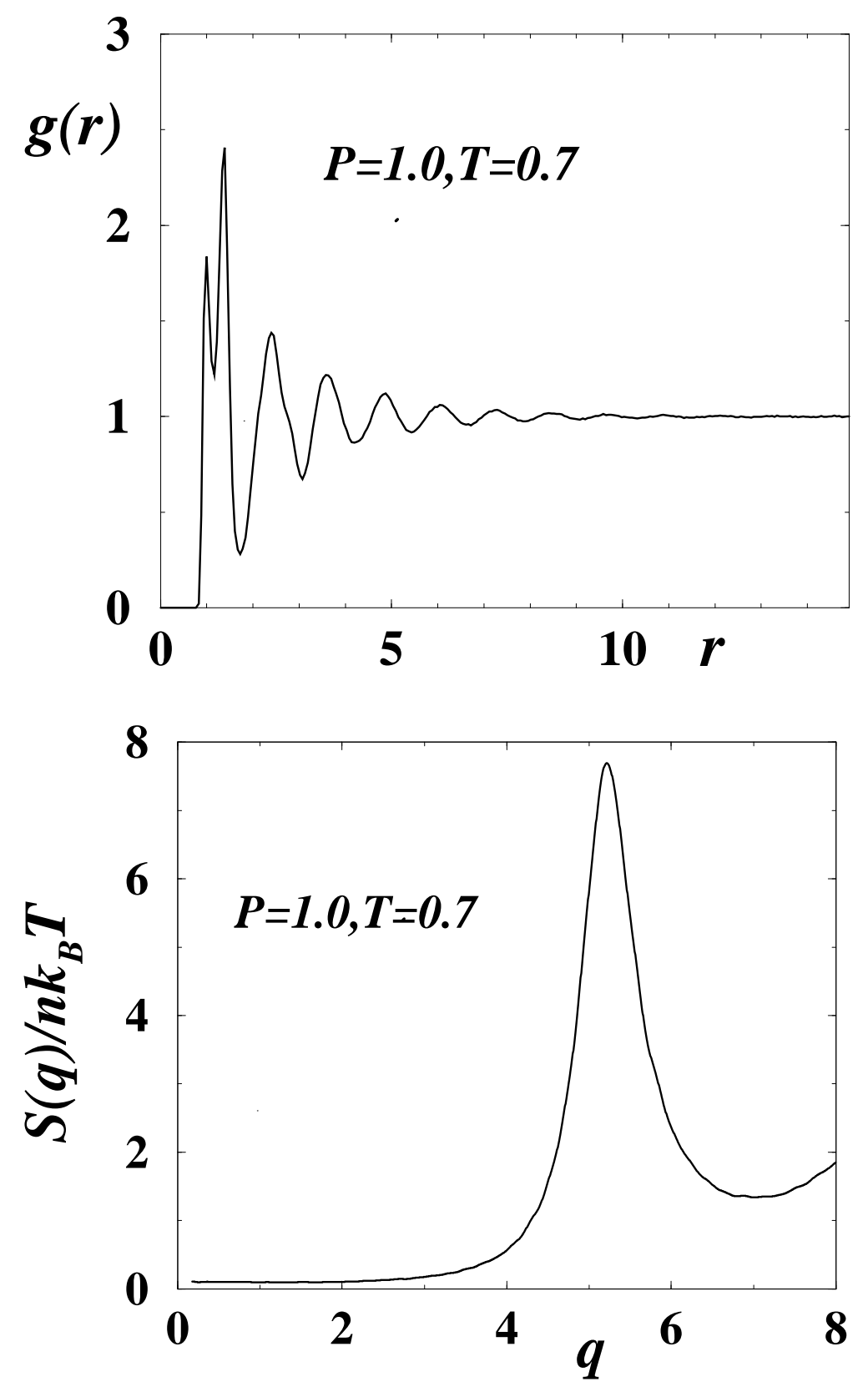

FIG. 11. Averaged pair distribution function for the smooth potential at $P=1.0, T=0.7$ (upper panel). The structure function (lower panel), multiplied by the factor $1 / n k_{B} T$, derived by integrating $g(r)$, where $k_{B}$ is the Boltzmann constant. The $q \rightarrow 0$ limit of this function gives $K_{T}$, which is around 0.1 in this case. 

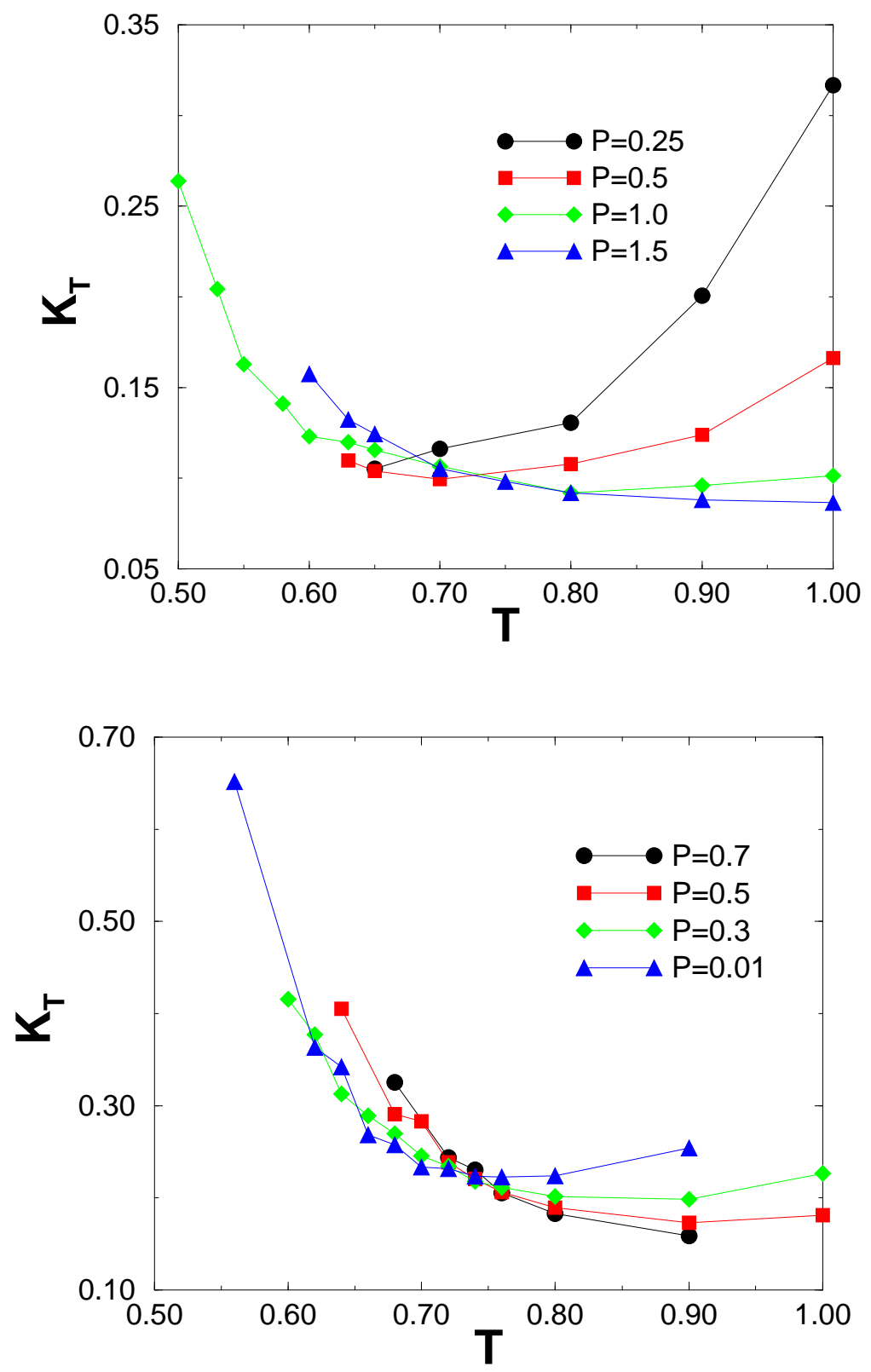

FIG. 12. Isothermal compressibility along isobars for the the continuous potential (left panel) and for the discrete potential (right panel). 